\title{
Komplexitätssteigerung medizinischer Entscheidungssituationen - Herausforderungen der Digitalisierung erkennen und gestalten
} Increasing complexity of medical decision-making situations recognising and shaping the challenges of digitalisation

\section{(ㅇ) (1) (요 $\ominus$}

Autoren

Stefanie Büchner ${ }^{1}$, Michael Marschollek ${ }^{2}$, Nilufar Foadi ${ }^{3}$

Institute

1 Institut für Soziologie und L3S Research Center, Leibniz Universität Hannover

2 Peter L. Reichertz Institut für Medizinische Informatik der Technischen Universität Braunschweig und der Medizinischen Hochschule Hannover

3 Klinik für Anästhesiologie und Intensivmedizin, Medizinische Hochschule Hannover

Schlüsselwörter

Digitalisierung, Entscheidung, Komplexität, hybrid, Organisation

Key words

Digitization, decision-making, complexity, hybrid, organisation

eingereicht $\quad 17.11 .2021$

akzeptiert $\quad 07.12 .2021$

online publiziert $\quad 28.01 .2022$

\section{Bibliografie}

Gesundh ökon Qual manag 2022; 27: 138-143

DOI 10.1055/a-1695-4507

ISSN $1432-2625$

(c) 2022. The Author(s). This is an open access article published by Thieme under the terms of the Creative Commons Attribution-NonDerivative-NonCommercialLicense, permitting copying and reproduction so long as the original work is given appropriate credit. Contents may not be used for commercial purposes, or adapted, remixed, transformed or built upon. (https://creativecommons.org/ licenses/by-nc-nd/4.0/)

Georg Thieme Verlag KG, Rüdigerstraße 14, 70469

Stuttgart, Germany

Korrespondenzadresse

Jun.-Prof. Dr. Stefanie Büchner

Leibniz Universität Hannover

Im Moore 21

30167 Hannover

Tel.: + $4915771567582^{n}$

s.buechner@ish.uni-hannover.de, buechner@|3s.de

\section{ZUSAMMENFASSUNG}

Die Chancen und Grenzen der Digitalisierung für medizinische Entscheidungssituationen werden bislang stark in Bezug auf die Potenziale einzelner Technologien und digitaler Tools diskutiert. Im Anschluss an soziologische Perspektiven, die medizinisches Entscheiden als sozial eingebettet und hybrid verstehen, zeigen wir zentrale strukturelle Herausforderungen der Digitalisierung in klinischen Entscheidungssituationen auf und entwickeln Handlungsempfehlungen für die Praxis. Gelingt es, strukturelle Herausforderung der Digitalisierung positiv zu bewältigen, eröffnen sich nachhaltige Möglichkeiten zur Verbesserung medizinischer Entscheidungssituationen durch Digitalisierung. Mit dieser integrativen Perspektive gelingt es, Engführungen auf einzelne Technologien und Idealisierungen von Entscheidungssituationen zu vermeiden, nichtintendierte Folgen zu antizipieren und Perspektiven für mittel- und langfristige Qualitätssteigerungen zu eröffnen.

\section{ABSTRACT}

The opportunities and limits of digitalisation for medical decision-making situations have been discussed heavily so far regarding to the potentials of single technologies and digital tools. Following sociological perspectives, which understand medical decision-making as socially embedded and hybrid, we show central structural challenges of digitalisation in clinical decision-making situations and develop recommendations for action for practice. If the structural challenges of digitalisation can be overcome positively, sustainable opportunities for improving medical decision-making situations through digitalisation will open up. With this integrative perspective, it is possible to avoid narrowing down to individual technologies and idealising decision-making situations, to anticipate unintended consequences and to open up perspectives for medium- and long-term quality improvements. 


\section{Einleitung}

In fast allen Entscheidungslagen sind in der Medizin mittlerweile digitale Daten und digitale Technologien involviert. Sie bestimmen die Entscheidungslage jedoch weder allein noch exklusiv [1]. In diesem Artikel widmen wir uns der Frage, welche strukturellen Herausforderungen die digitale Transformation für medizinische Entscheidungssituationen bereithält und welche Handlungsempfehlungen sich daraus ableiten lassen.

Unser Lebens- und Arbeitsalltag nimmt immer stärker hybride Züge an, angefangen von unserer mittlerweile engen Verbindung mit Smartphones [2] bis zu hochspezialisierten Prozessen wie der Sepsis Einschätzung [3]. Unter ,hybriden‘ Phänomenen versteht man in der Soziologie allgemein die Durchdringung und das Ineinandergreifen unterschiedlicher Elemente. In Bezug auf Entscheidungssituationen meint das Attribut „hybrid“, dass unterschiedliche Medien, Artefakte, menschliche und maschinelle Akteure und damit auch eine Vielzahl an Wissensformen an Entscheidungen beteiligt sind [4], [5]. ' Entsprechend sind die meisten Entscheidungssettings hybrid, statt dass sie sich sinnvoll binär in „digital“ oder „nicht digital“ unterscheiden lassen.

Neben Hybridität ist der Alltag medizinischen Entscheidens erheblich durch seine Organisiertheit geprägt: Klinisches Entscheiden findet nie idealisiert und freigestellt von Handlungsdruck statt, sondern ist immer organisiert: Personaldecken, Schichtpläne und Bettenauslastungen sind Beispiele für die Bedeutung des Kontextes „Organisation“ für hybrides medizinisches Entscheiden [1], [10], [11], [12].

Wir greifen im Folgenden exemplarisch vier zentrale Bereiche heraus, in denen Digitalisierung zu einer Zunahme an Komplexität in medizinischen Entscheidungssituationen und damit zum Entstehen neuer Herausforderungen führt. Ein integriertes Verständnis medizinischen Entscheidens als hybriden Prozess kann dazu beitragen, Technikentwicklung mit größerer Sensibilität für die soziale Einbettung von Technologien zu gestalten.

\section{Daten und Informationen im klinischen}

\section{Arbeitsumfeld - Integration und reflektierte Bewertung von Informationen als \\ Herausforderung}

Das Vorhandensein einer großen Bandbreite an Daten ermöglicht grundsätzlich eine präzise Ausrichtung der medizinischen Behandlung auf den individuellen Patienten im Sinne der personalisierten Medizin [13]. Informationsquellen liegen allerdings meist nicht gleichzeitig vor und sind, was besonders bei der Koexistenz von Papier- und digitalen Akten der Fall ist, zumeist nicht synchronisiert. Es fließen außerdem eine Vielzahl von Kontextinformationen, etwa die Eignung einer Therapie für ein bestimmtes soziales Umfeld, in die Entscheidungsfindungen ein. Hinzu kommt, dass gerade bei komplexen digitalen Anwendungen wie Diagnoseunterstützungs-

\footnotetext{
Weitere Kernkonzepte wie das der Soziomaterialität [6], [7] oder der soziotechnischen Systeme [8], [9] sensibilisieren in ähnlicher Weise für die Bedeutung der engen sozialen Einbettung von Technologien. Weil es uns an dieser Stelle nicht um eine konzeptuelle Diskussion geht, sondern um die Diskussion der Implikationen eines solchen erweiterten Fokus auf Digitalisierung und die Formulierung von Handlungsempfehlungen, konzentrieren wir uns hier auf das Konzept der Hybridität.
}

systemen ein Nachvollzug und eine Bewertung der eingeschlossenen und ausgeschlossenen Studien im Einzelfall kaum mehr möglich ist. Dieses Spannungsfeld wird unter dem Schlagwort der Opazität von Algorithmen diskutiert [14].

Gerade im Angesicht wachsender digitaler Datenmengen wird die Markierung relevanter Informationen immer wichtiger. Hierbei ist wichtig zu erkennen, dass gerade Medienbrüche Signalfunktionen übernehmen können [15], [16]. Eine auf Papier geschriebene Notiz, die möglicherweise von Relevanz für die weitere Behandlung ist, ein mündlich betonter Hinweis über den Gang der Behandlung bei der Übergabe, die auf Visite vorgetragene vorsichtige Sorge über unklare Werte - all dies sind wichtige soziale Markierungen, die gerade bei einer hohen Dichte an digitalen Informationen bedeutsam werden können.

In einem hybriden und eingebetteten Entscheidungsprozess eröffnen sich durch diesen Zugewinn nicht nur neue Möglichkeiten, sondern auch strukturelle Herausforderungen: Welche Informationen werden im medizinischen Entscheidungsprozess aus welchen Quellen erhoben, aus heterogenen Quellen zusammengeführt und auf welche Weise beurteilt?

\section{Handlungsempfehlungen}

Es bedarf seitens medizinischer Fachkräfte ein generelles Bewusstsein darüber, wie sachdienliche Informationen aus digitalen Daten gewonnen werden können [17]. Hierzu benötigen Ärzte und Pflegekräfte ein Verständnis der Funktionsprinzipien digitaler Systeme wie der elektronischen Patientenakte und weiterer digitaler Technologien [18].

Zugleich benötigt das medizinische Personal grundlegende Kenntnisse und ein Bewusstsein darüber, inwieweit die Art und Weise, auf welche Ärzte und Pflegekräfte Daten pflegen und ihre Dokumentation durchführen, die Qualität von medizinischen Daten beeinflusst [19], [20]. Hierbei ist sowohl auf der Seite der digitalen Tools als auch bei der Durchführung der Dokumentation durch das medizinische Personal eine Struktur nötig, die eine klare und eindeutige Verständlichkeit gewährleistet. Es gilt zu vermeiden, dass Daten mehrdeutig interpretierbar sind.

Das Wissen um Dokumentationspraktiken sollte über die Ebene des „tacit knowledge“ [21], [22] hinaus explizit gemacht und reflektiert werden. Zugleich sollten Algorithmen eines angewendeten digitalen Entscheidungsunterstützungssystems erklären, warum das System zu einer bestimmten Entscheidung rät. Im Falle eines SIRS-Systems sollte z. B. erkennbar sein, welche Regel aktiv wurde und welche Werte in Kombination abnormal seien [23].

Um eine hohe Datenqualität aufrechtzuerhalten, benötigt es, solange dies noch nicht automatisiert durch die digitalen Systeme erfolgt, eine aktive Überprüfung, inwieweit eine den Qualitätskriterien entsprechende standardisierte Datenerfassung vorliegt. Diese Arbeit, diese zusätzliche „data work“ [24], [25] gilt es sichtbar zu machen und in der Planung von Arbeitsprozessen einzukalkulieren [26].

Es gilt, offen für Informationslagen aus unterschiedlichen Medien und in unterschiedlichen Dokumentationsgraden zu bleiben [27], [28]. Das Bewusstsein über die Vielfalt und den Wert unterschiedlicher Informationsquellen und -träger sollte im medizinischen Alltag geschärft werden. 
Fehler-Paradoxie - Fehlervermeidung und Fehlerentstehung in hybriden Entscheidungssituationen

Digitalisierungsprojekte zielen oft auf Fehlerreduktion und Fehlervermeidung ab. Zwar dient data work in spezifischen Bereichen dem Ziel einer besseren Patientenversorgung. Dies ist jedoch keineswegs per se der Fall. Hier existieren strukturelle Spannungen zwischen Dokumentationsarbeit und der Arbeit am Patienten [29]. Diese strukturellen Herausforderungen anzuerkennen, heißt, sich dafür zu öffnen, dass es „good organizational reasons for bad medical records“ [30] geben kann. Gerade bei steigenden Erwartungen an Datenqualität im Zuge der Nachnutzung von Behandlungsdaten ist hier mit Friktionen zu rechnen. So weist eine Studie im hochdigitalisierten dänischen Gesundheitswesen darauf hin, dass gestiegene Erwartungen an Datenpflege für Professionelle zunehmend „meaningless work“ [31] produziert. Anstelle der Unterstützung der klinischen Entscheidungsfindung führt dies zu einer erheblichen Bürokratisierung von Behandlungsprozessen.

Ein essenzieller Bestandteil der Fehlervermeidung besteht nicht zuletzt auch in Konfigurationsarbeit, die in soziotechnischen und hybriden Konstellationen entsteht. Situatives Handeln prägt den klinischen Alltag in der Medizin. Hierdurch ergibt sich, dass Formalstrukturen, die in digitalen Systemen hinterlegt sind, nicht in jedem Fall eingehalten werden können [32]. Eine enge Einbindung dieser Systeme erzeugt neue Herausforderungen. Entsprechend muss situativ abweichendes, aber nötiges Handeln neue Wege finden, trotzdem realisiert zu werden [33].

Das „bottleneck“ menschlicher Aufmerksamkeit ist als ein limitierender Faktor seit langem bekannt [34], [35]. Eine Zunahme an Warnmeldungen durch digitale Tools führt nicht notwendiger Weise zu den intendierten Effekten. Betrachtet man die große Zahl an visuellen und akustischen Alarmen, die von patientennahen Geräten, beispielsweise im intensivstationären Bereich, ausgehen, so wird erkennbar, dass das medizinische Personal im Laufe der Zeit gegenüber den Alarmen desensibilisiert. Es zeigt sich eine „AlarmFatigue“, die schlimmstenfalls die Patientensicherheit gefährden kann [36]. Wenn digitale Tools Warnmeldungen automatisiert in Patientendatensysteme ausleiten, können durch dieses Capturing [37], d. h. durch das gleichzeitige Erheben und Speichern der Information, Alarmierungen retrospektiv nachvollzogen werden und nicht nur informelle, sondern auch formale Verantwortungsfragen mit sich bringen.

\section{Handlungsempfehlungen}

Die Art und Weise, wie und von wem mit welchen zeitlichen Ressourcen digitale Daten erfasst und verarbeitet werden, sollte bei der Aufstellung von Qualitätskriterien für eine standardisierte Dokumentation mitberücksichtigt werden. Für eine Qualitätssteigerung prozesserzeugter Daten sind zweifelsohne vermehrte Investitionen nötig. Wenn nicht entsprechende personelle Kapazitäten vorhanden sind, bedarf es neuer Stellen beispielsweise für spezialisierte Data Stewards [38], [24]. Idealerweise geschieht die Daten Kuration eingebettet in die laufenden Dokumentationsprozesse. Dabei sollten (inter)nationale Vorgaben berücksichtigt werden, um eine einheitliche Semantik sicherzustellen.
Bei der Implementierung neuer Dokumentationssysteme sind quantitativ und qualitativ begleitende Untersuchungen nötig, um Fehlentwicklungen wie der Bürokratisierung des Arbeitsalltags vorzubeugen bzw. sie zumindest frühzeitig zu erkennen.

Das medizinische Personal sollte darin geschult werden, wie es zur Aufrechterhaltung einer hohen Datenqualität beitragen kann und wie Datenambiguität vermieden werden kann. Hierbei gilt es, strukturelle Spannungen anzuerkennen und thematisierbar zu machen, um die angespannte Fachkräftesituation nicht zu verschärfen und nachhaltig personelle Ressourcen zu sichern.

Psychosoziale Zusammenhänge, die assoziiert sind mit der begrenzten menschlichen Aufmerksamkeit, sollten beim Design von Frühwarnsystemen und ihrer Testung in Studiendesigns konsequent mitberücksichtigt werden. Unter Einbeziehung des medizinischen Personals könnte eine für das jeweilige klinische Arbeitsumfeld passende und überschaubare Anzahl an Alarmen sowie eine angemessene sensorische Form gewählt werden. Je besser das Personal mit den Funktionsprinzipien der angewendeten Systeme vertraut ist, umso suffizienter können die digitalen Systeme in die klinischen Abläufe integriert werden [39].

Ärzten sollten darin geschult werden, wie die Art und Weise des Sammelns relevanter Informationen und soziale und kontextbezogene Faktoren bei Denkprozessen Einfluss auf den Entscheidungsprozess nehmen [40], [41]. Einflüsse impliziten Wissens als „analoger Bestandteil“ bei der Informationsakquise sollten in der medizinischen Ausbildung explizit thematisiert werden.

\section{Die Ressource „Zeit“ - Effizienzgewinn versus hohen Zeitaufwand durch Digitalisierung}

Jede Minute weniger, in der medizinische Fachkräfte dokumentieren, Eingaben tätigen oder Klicks in digitalen Systemen verrichten müssen, ist eine Minute mehr für den direkten Patientenkontakt [42]. Die reale Arbeitssituation ist in vielen klinischen Einrichtungen dadurch geprägt, dass Ärzte und Pflegekräfte mehr Zeit mit Dokumentation verbringen als im direkten Patientenkontakt [43], [44].

Parallel dazu hat sich ein Wandel der Praktiken des Informierens bei Patienten vollzogen, der sich auch auf die zeitlichen Ressourcen des medizinischen Personals auswirkt. Durch wachsendes Interesse an gesundheitsbezogenen Informationen recherchieren zunehmend mehr Patienten eigeninitiativ umfassender über ihre Krankheiten [45]. Hier zeigen sich auch soziostrukturell unterschiedliche Nutzerverhalten und soziokulturell unterschiedliche Kommunikationsverhalten, was Ärzten eine zusätzliche Sensibilisierung für die veränderte Arzt-Patienten-Kommunikation in der digitalen Ära abverlangt [46]. Der „vorinformierte“ Patient zieht dabei eine Erweiterung des Aufgabenspektrums von Ärzten nach sich [47]. Vorinformationen müssen im Gespräch eruiert, kontextualisiert und oft auch korrigiert werden, um zu einem geteilten Krankheits- und Behandlungsverständnis zu gelangen. Diese durch Digitalisierung ermöglichten veränderten Informationslagen erzeugen neue Kommunikationsbedarfe, die nicht nur fachlich, sondern auch zeitlich bewältigt werden müssen. 


\section{Handlungsempfehlungen}

Für die Stärkung des Empowerments von Patienten sind digital breit zugängliche Informationen nützlich [48]. Zusätzliche ärztliche Beratungszeit des „vorinformierten Patienten“ sollte im ärztlichen Aufgabenspektrum strukturell allokiert und abgebildet werden. Es gilt, die veränderte ärztliche Rolle in Bezug auf die Arzt-PatientenKommunikation in einer digitalisierten Gesellschaft in der medizinischen Aus- und Weiterbildung zu adressieren [49].

Nicht nur für die Versorgungsqualität und Zufriedenheit von Patienten und dem langfristigen Wohlbefinden und der Zufriedenheit des medizinischen Personals ist es erstrebenswert, dass die im klinischen Alltag verwendeten digitalen Anwendungen die Zeit des medizinischen Personals so wenig wie möglich beanspruchen [50], [51]. Die Art und Weise, wie Arbeitsabläufe und die Interaktion mit digitalen Werkzeugen individuell gestaltet werden, unterliegt innerhalb des medizinischen Personals einer Varianz. Dies sollte bei der Entwicklung von zukünftigen digitalen Systemen Berücksichtigung finden [52]. Auch aus ökonomischer Sicht ist es sinnvoll, dass die Zeit für Dokumentation so überschaubar wie möglich gehalten wird, geht doch ein hoher Dokumentationsaufwand mit erhöhten Personalkosten einher [53]. Ein intelligenter, auf die Bedürfnisse der Anwender angepasster Einsatz digitaler Technologien, beispielsweise das Einbinden von Tonaufnahmen und digitaler Spracherfassung, sollte dazu beitragen, dass der direkte Patientenkontakt wieder verstärkt in den Fokus gerückt wird.

\section{Diversere Daten, passgenaue digitale Lösungen?}

Von digitalen Tools profitieren Patienten keinesfalls gleich: Werden Mustererkennungen für Hautkrebs vorwiegend auf weißer Haut trainiert, können sich nichtweiße Patienten auf systematisch weniger verlässliche Entscheidungsunterstützungssysteme verlassen. Obgleich wir theoretisch und praktisch über Möglichkeiten der geschlechtersensiblen Behandlung von Erkrankungen verfügen, fungieren in vielen Bereichen nach wie vor Männer als „generische Patienten“ bzw. als Ausgangspopulation [54]. Dieser Umstand sensibilisiert für die immense Bedeutung von Routinen und Konventionen in hybriden Entscheidungssituationen.

Entscheidungslagen werden hier durch Digitalisierung keineswegs ausschließlich vereinfacht, sondern gewinnen an Komplexität. Hier entstehen neue Fragestellungen wie die, auf welche Patientengruppe hin ein digitales Tool optimiert wurde und wie Fehlerquoten zwischen unterschiedlichen Patientengruppen schwanken.

\section{Handlungsempfehlung}

Im Zuge der medizinischen Aus- und Weiterbildung wird eine differenzierte Reflexion über den antizipierten „User“/„Patienten“ nötig. Diese Reflexion legt Normalitätsannahmen offen, die erst dadurch bearbeitbar werden.

Es bedarf parallel zur Optimierung digitaler Tools vermehrter Initiativen für die Entwicklung diversitätssensibler digitaler Werkzeuge. Zur Stärkung diversitätssensibler Forschungen gilt es, Daten stärker zu kontextualisieren und damit strukturelle Einseitigkeiten in der Forschungspraxis offen zu legen und zu begegnen.

\section{Fazit und Ausblick - strukturelle Herausforderungen von sozial eingebetteten und hybriden Entscheidungslagen erkennen und verantwortlich gestalten}

Ein systematischerer Einbezug soziologischer Perspektiven für die Analyse und Gestaltung von „digital health“ kann zukünftig IT-Fachdisziplinen in der Medizin bereichern [55]. Die Chance der gesamtheitlichen soziotechnischen Betrachtung besteht darin, Digitalisierung nicht einseitig, sondern unter systematischer Berücksichtigung ihrer strukturellen Effekte, etwa der hier herausgestellten Komplexitätssteigerung, zu reflektieren und damit in der Organisationsgestaltung und Medizinausbildung berücksichtigen zu können. Die Entwicklungschance besteht darin, die mögliche Vielfalt der Umgangsweisen mit den dargestellten Herausforderungen sichtbar zu machen und diejenigen zu fördern, die die Qualität der Patientenversorgung mittel- und langfristig steigert. Eine integrierte Entwicklung verbindet hier Organisationswandel und Digitalisierungsstrategien.

Mittel- und langfristig gilt es, diese sozialen und hybriden Einbettungen medizinischen Entscheidens systematisch und interdisziplinär zu untersuchen. Aufgrund ihrer Kontextsensibilität eignen sich im besonderen Maße qualitative Methoden wie ethnografische Begleitungen, Interviews und Gruppendiskussionen. Auch eine Stärkung partizipativer Verfahren der Technologieentwicklung und -einführung kann helfen, diese strukturellen Herausforderungen sichtbar zu machen und verantwortlich zu gestalten. Digitalisierung ist in diesem Sinne im Idealfall nicht verkürzt auf Technik, sondern wird als nachhaltige soziotechnische Innovation sichtbar.

\section{Interessenkonflikt}

Die Autorinnen/Autoren geben an, dass kein Interessenkonflikt besteht.

\section{Literatur}

[1] Büchner S. Zum Verhältnis von Digitalisierung und Organisation. Zeitschrift Für Soziologie 2018; 47: 332-348. doi:10.1515/ zfsoz-2018-0121

[2] Mazmanian M. Worker/Smartphone Hybrids: The Daily Enactments of Late Capitalism. Management Communication Quarterly 2019; 33: 124-132. doi:10.1177/0893318918811080

[3] Maiers C. Analytics in action: users and predictive data in the neonatal intensive care unit. Information, Communication \& Society 2017; 20: 915-929. doi:10.1080/1369118X.2017.1291701

[4] Edwards PN. (2018). We Have Been Assimilated: Some Principles for Thinking About Algorithmic Systems. In: Schultze U, Aanestad M, Mähring M. et al., eds. Living with Monsters? Social Implications of Algorithmic Phenomena, Hybrid Agency, and the Performativity of Technology 2018. Cham: Springer; 2018: 19-27. doi:10.1007/978-3030-04091-8_3

[5] Suchman L. Human-Machine Reconfigurations - Plans and Situated Actions. 2nd ed.Cambridge: Cambridge University Press; 2007. doi:10.1017/СBO9780511808418 
[6] Orlikowski W]. Sociomaterial Practices: Exploring Technology at Work. Organization Studies 2007; 28: 1435-1448. doi: $10.1177 / 0170840607081138$

[7] Orlikowski W]. The sociomateriality of organisational life: Considering technology in management research. Cambridge Journal of Economics 2010; 34: 125-141. doi:10.1093/cje/bep058

[8] Rammert W. Technik - Handeln - Wissen: Zu einer pragmatistischen Technik- und Sozialtheorie. 2. Aufl.Wiesbaden: Springer VS; 2016. doi:10.1007/978-3-658-11773-3

[9] Rammert W, Schubert C, Hrsg.. Technografie: Zur Mikrosoziologie der Technik. Frankfurt am Main: Campus-Verlag; 2006359338227X

[10] Strauss AL, Fargerhaugh S, Suczek B et al. Social Organization of Medical Work. Chicago: University of Chicago Press; 1985. doi:10.4324/9781315129679

[11] Vogd W. Ärztliche Entscheidungsfindung im Krankenhaus - Komplexe Fallproblematiken im Spannungsfeld von Patienteninteressen und administrativ-organisatorischen Bedingungen. Zeitschrift Für Soziologie 2004; 33: 26-47. doi:10.1515/zfsoz-2004-0102

[12] Vogd W. Management im Krankenhaus - Eine Fallstudie zur Bearbeitung der Innen- und Außenspannungen unter den Bedingungen des deutschen DRG-Systems. Berliner Journal Für Soziologie 2017; 27: 93-121. doi:10.1007/s11609-017-0332-8

[13] Schüssler-Fiorenza RSM, Contrepois K, Moneghetti KJ et al. A longitudinal big data approach for precision health. Nat Med 2019; 25 : 792-804. doi:10.1038/s41591-019-0414-6

[14] Burrell J. How the machine 'thinks'. Understanding opacity in machine learning algorithms. Big Data \& Society 2016; 3: 1-12. doi:10.1177/2053951715622512

[15] Meum T. "Lost in translation": The challenges of seamless integration in nursing practices. International Journal of Medical Informatics 2013; 82: 200-208. doi:10.1016/j.jijmedinf.2012.11.012

[16] Wadmann S, Hoeyer K. Dangers of the digital fit: Rethinking seamlessness and social sustainability in data-intensive healthcare. Big Data \& Society 2018; 5: 1-13. doi:10.1177/2053951717752964

[17] Jimenez G, Spinazze P, Matchar D et al. Digital health competencies for primary healthcare professionals: A scoping review. Internationa Journal of Medical Informatics 2020; 143: 104260. doi:10.1016/j. ijmedinf.2020.104260

[18] Foadi N, Koop C, Behrends M. Welche digitalen Kompetenzen braucht der Arzt? Deutsches Ärzteblatt 2020; 117: A 596-600

[19] Jacob A, Raj R, Alagusundaramoorthy $S$ et al. Impact of patient load on the quality of Electronic Medical Record documentation. Journal of Medical Education and Curricular Development 2021; 8: 1-6. doi:10.1177/2382120520988597

[20] Lorenzetti DL, Quan H, Lucyk K et al. Strategies for improving physician documentation in the emergency department: a systematic review. BMC Emergency Medicine 2018; 18: 36. doi:10.1186/s12873-0180188-z

[21] Hadjimichael D, Tsoukas H. Toward a Better Understanding of Tacit Knowledge in Organizations: Taking Stock and Moving Forward. The Academy of Management Annals 2019; 13: 672-703. doi:10.5465/ annals.2017.0084

[22] Polanyi M. The tacit dimension. London: Routledge \& Kegan; 1967

[23] Holzinger A, Langs $G$, Denk $H$ et al. Causability and explainability of artificial intelligence in medicine. Wiley Interdisciplinary Reviews: Data Mining Knowledge Discovery 2019; 9: e1312. doi:10.1002/widm.1312

[24] Pine KH, Bossen C. Good organizational reasons for better medical records: The data work of clinical documentation integrity specialists. Big Data \& Society 2020; 7: 1-13. doi:10.1177/2053951720965616

[25] Ruckenstein M, Schüll ND. The Datafication of Health. Annual Review of Anthropology 2017; 46: 261-278. doi:10.1146/annurevanthro-102116-041244
[26] Lucas B, Mathieu SC, Pliske G et al. The impact of a qualified medical documentation assistant on trauma room management. European Journal of Trauma an Emergency Surgery 2020. doi:10.1007/ s00068-020-01513-y

[27] Shortliffe EH, Sepúlveda M]. Clinical decision support in the era of artificial intelligence. JAMA 2018; 320: 2199-2200. doi:10.1001/ jama.2018.17163

[28] Harrison MI, Koppel R, Bar-Lev S. Unintended consequences of information technologies in health care-an interactive sociotechnical analysis. Journal of the American Medical Informatics Association 2007; 14: 542-549. doi:10.1197/jamia.M2384

[29] Büchner S. Fallsoftware als digitale Dokumentation - Zur Unterscheidung einer Arbeits- und Organisationsperspektive auf digitale Dokumentation. In Neuhaus L, Käch O, Hrsg.. Bedingte Professionalität - Professionelles Handeln im Kontext von Institution und Organisation. Weinheim: Beltz Juventa; 2018: 240-269

[30] Garfinkel H. 'Good' organisational Reasons for 'bad' clinical Records. In: Garfinkel H, Hrsg.. Studies in Ethnomethodology. New York: Prentice-Hall; 1967: 186-207

[31] Hoeyer K, Wadmann S. 'Meaningless work': How the datafication of health reconfigures knowledge about work and erodes professional judgement. Economy and Society 2020; 49: 433-454. doi:10.1080/03 085147.2020 .1733842

[32] Kühl S. Brauchbare Illegalität. Vom Nutzen des Regelbruchs in OrganisationenFrankfurt am Main: Campus Verlag; 2020

[33] Pine KH, Mazmanian M. Artful and Contorted Coordinating: The Ramifications of Imposing Formal Logics of Task Jurisdiction on Situated Practice. Academy of Management Journal 2017; 60: 720-742. doi:10.5465/amj.2014.0315

[34] March JG, Olsen JP, Hrsg.. Ambiguity and Choice in Organizations. Oslo: Scandinavian University Press; 1994

[35] Ocasio W. Situated attention, loose and tight coupling, and the garbage can model. In: Lomi A, Harrison RJ, Hrsg.. Garbage can model of organizational choice. Looking forward at fortyBingley: Emerald; 2012: 293-317. doi:10.1108/S0733-558X(2012)0000036014

[36] Lorenz B, Peters J, Frey UH. Alarm-Fatigue - wieviel Alarm verträgt der Mensch? Anästhesiologie Intensivmedizin Notfallmedizin Schmerztherapie 2017; 52: 564-570. doi:10.1055/s-0042-118618

[37] Agre PE. Surveillance and capture: Two models of privacy. The Information Society 1994; 10: 101-127. doi:10.1080/01972243.1994. 9960162

[38] Bossen C, Chen Y, Pine KH. The emergence of new data work occupations in healthcare: The case of medical scribes. International Journal of Medical Informatics 2019; 123: 76-83. doi:10.1016/j. ijmedinf.2019.01.001

[39] Heller AR, Mees ST, Lauterwald B et al. Detection of deteriorating patients on surgical wards outside the ICU by an automated MEWS-based early warning system with paging functionality. Annals of Surgery 2020; 271: 100-105. doi:10.1097/SLA.0000000000002830

[40] Gäbler M. Denkfehler bei diagnostischen Entscheidungen. Wiener Medizinische Wochenschrift 2017; 167: 333-342. doi:10.1007| s10354-017-0570-6

[41] Blumenthal-Barby JS, Krieger H. Cognitive biases and heuristics in medical decision making: a critical review using a systematic search strategy. Medical Decision Making 2015; 35: 539-557. doi:10.1177/02 72989X14547740

[42] Lown BA, Rodriguez D. Commentary: Lost in translation? How electronic health records structure communication, relationships, and meaning. Academic medicine: journal of the Association of American Medical Colleges 2012; 87: 392-394. doi:10.1097/ ACM.0b013e318248e5ae 
[43] Arndt BG, Beasley JW, Watkinson MD et al. Tethered to the EHR: primary care physician workload assessment using EHR event log data and time-motion observations. The Annals of Family Medicine 2017; 15: 419-426. doi:10.1370/afm.2121

[44] Warner JL, Smith J, Wright A. It's time to wikify clinical documentation: how collaborative authorship can reduce the burden and improve the quality of the Electronic Health Record. Academic medicine: journal of the Association of American Medical Colleges 2019; 94: 645-650. doi:10.1097/ACM.0000000000002613

[45] Rodriguez JA, Clark CR, Bates DW. Digital health equity as a necessity in the 21 st century cures act era. JAMA 2020; 323: 2381-2382. doi:10.1001/jama.2020.7858

[46] Mertz L. Medical care in the digital era. IEEE Pulse 2021; 12: 2-5. doi:10.1109/MPULS.2021.3078600

[47] Erikainen S, Pickersgill M, Cunningham-Burley S et al. Patienthood and participation in the digital era. Digit Health 2019; 5: 2055207619845546. doi:10.1177/2055207619845546

[48] Calvillo J, Román I, Roa LM. How technology is empowering patients? A literature review. Health Expectations 2015; 18: 643-652. doi:10.1111/hex.12089

[49] Foadi N, Koop C, Mikuteit M et al. Defining learning outcomes as a prerequisite of implementing a longitudinal and transdisciplinary curriculum with regard to digital competencies at Hannover Medical School. Journal of Medical Education and Curricular Development 2021; 8: 1-9. doi:10.1177/23821205211028347
[50] Schmitt-Sausen N. Digitale Medizin: Ärzte müssen eingebunden werden.Deutsches Ärzteblatt 2019; 116: A-630/B-516/C-508

[51] Shachak A, Reis $S$. The impact of electronic medical records on patient-doctor communication during consultation: A narrative literature review. Journal Evaluation Clinical Practice 2009; 15: 641-649. doi:10.1111/j.1365-2753.2008.01065.x

[52] Neri PM, Redden L, Poole $S$ et al. Emergency medicine resident physicians' perceptions of electronic documentation and workflow: a mixed methods study. Applied Clinical Informatics 2015; 6: 27-41. doi:10.4338/ACI-2014-08-RA-0065

[53] Himmelstein DU, Wright A, Woolhandler S. Hospital computing and the costs and quality of care: a national study. The American Journal of Medicine 2010; 123: 40-46. doi:10.1016/j.amjmed.2009.09.004

[54] Cirillo D, Catuara-Solarz S, Morey C et al. Sex and gender differences and biases in artificial intelligence for biomedicine and healthcare. NPJ Digital Medicine 2020; 3: 81. doi:10.1038/s41746-020-0288-5

[55] Henwood F, Marent B. Understanding digital health: Productive tensions at the intersection of sociology of health and science and technology studies. Sociology of Health \& Illness 2019; 41: 1-15. doi:10.1111/1467-9566.12898 